\title{
MODWT, PCA and Decision Tree based Fault diagnosis of Gear
}

\author{
MANSI ${ }^{1, *}$,Sukhdeep S. DHAMI ${ }^{1}$ and VANRAJ ${ }^{2}$ \\ ${ }^{1}$ Department of Mechanical Engineering, National Institute of Technical Teachers Training \\ \& Research, Chandigarh, India \\ ${ }^{2}$ Labrose Ayurveda OPC Private Limited, Haryana-India
}

DOI: $10.51201 /$ JUSST/21/07161

\begin{abstract}
http://doi.org/10.51201/JUSST/21/07161
Abstract: A gearbox is an important power transmission equipment. Its maintenance is a top requirement because it is prone to a variety of failures. For gearbox fault diagnosis, techniques such as vibration monitoring have been widely used. Also, when it comes to machine Condition monitoring and fault diagnostics, feature extraction is the crucial step. For a classifier to perform accurately, it must have the appropriate discriminative information or features. Hence, this paper proposes a signal processing methodology based on Maximal overlap discrete wavelet transform (MODWT) and a dimensionality reduction technique i.eprincipal component analysis (PCA) to reduce the dimensionality of the feature space and obtain an ideal subspace for machine fault classification. Firstly, the raw vibration signature is denoised with the help of a state-of-the-art MODWT signal processing technique to identify the hidden fault signatures. Then various traditional statistical features are extracted from this denoised signal. These multi-dimensional features are then processed with PCA and further, the Decision Tree is used for fault classification. Performance comparison of the proposed method with traditional raw analysis and without application of PCA is presented and the proposed method outperforms at every level.
\end{abstract}

Keywords: Condition monitoring, Decision tree, Feature extraction, Gears, MODWT, PCA

\section{INTRODUCTION}

The gearbox is an essential component of industrial machines as it is responsible for transferring power. Gears are subjected to harsh conditions such as excessive torque, lack of lubrication, and installation issues, etc [1]. As a result of this, they are prone to several breakdowns so its maintenance is the most important criteria. According to statistics, gear faults account for 80 percent of failures in mechanical transmission systems and around 10 percent of rotating machinery failures [2]. To ensure the machine component's long life along with its safety and reliability, condition monitoring (CM) is the foremost step [3]. Therefore, gear condition monitoring is critical to make sure that the system's productivity and efficiency are not compromised. By successfully implementing condition monitoring routines, the machine can function at its maximum capacity without having to stop for inspections at predetermined intervals.

Vibration analysis is a well-established technique for fault diagnosis and condition monitoring of the mechanical component [4,5]. Vibration analysis provides extensive information about the machinery's operational conditions. The usual vibration of the machine is altered when the fault occurs in it. So, the vibration signatures of rotating parts such as gears provide a strong indication of its health decline and give timely indications when monitored [6].Vibration monitoring entails gathering data, processing it, and making decisions on how to proceed with maintenance. The vibration data is collected using an accelerometer that is mounted to the gearbox. Because vibration data is of the waveform type, it can be subjected to time-domain, frequency-domain, and time-frequency analysis [7]. The three primary components of fault diagnosis are characteristic signal recognition by utilizing 
proper signal processing techniques, feature extraction, and fault classification.

Non-stationary vibration signals of faulty gears, along with useful information contain noise that makes it quite difficult to identify weak fault transients and separate them. As a result, for this challenge, denoising, an efficacious signal processing approach should be implemented. The time-frequency distribution depicts the energy distribution of the signal for two-dimensional space (time and frequency) and provides a better description for nonstationary signals [8]. The most often used time-frequency strategies are the Short-Time Fourier Transform (STFT) [9,10], Hilbert-Huang Transform (HHT) [11,12], Wigner Ville Distribution (WVD) [13,14], and Wavelet Transform (WT) [15,16]. Among all the mentioned strategies, Wavelet transform (WT) has been considered a useful approach for analyzing non-stationary signals [17]. It has demonstrated its immense power in condition monitoring and fault diagnosis of mechanical equipment due to its multi-resolution analysis property, which facilitates finding weak fault features from noisy signals $[18,19]$. Discrete wavelet transform (DWT) is a popular tool for extracting time-frequency features in condition monitoring and fault diagnostics [20-22]. But, this has certain disadvantages which include the sample size must be exactly two (down-sampling) $[23,24]$, and the values that are downsampled at each level of decomposition are discarded by DWT. The Maximal overlap discrete wavelet transform (MODWT) is an improved variant of the DWT, which resolves its constraints such as downsampling and sample size [25,26].

Any indicators derived from measurements via signal processing to improve the fault detection process are referred to as features. Feature extraction is a crucial stage in the fault detection process. When the feature extraction is not correct or insufficient, inaccurate classification will certainly result. For the fault diagnosis of large-scale complicated machinery, the most vital step is to extract the effective fault characteristic information from their highly dynamic signals [27]. To collect the diagnostic information, as many features as feasible can be computed. However, which feature to be used is usually subjective, resulting in instances where multiple features may provide the same information or some may provide no meaningful insights at all. The processing of these redundant features may increase the learning cost and have an impact on the condition monitoring system's real-time application; hence dimensionality reduction is required. It's crucial for the accurate classification of faults. The PCA (Principal Component Analysis) [28] approach is an ideal dimension reduction methodology that uses the minimum mean square error as a criterion. In other words, the transformed data from the original data by utilizing the principal component analysis approach will retain the majority of the original data information. This method analyses the data using second-order statistics (namely, the corresponding covariance matrix) and also extracts each redundant feature component. It solves the characteristic equation and selects the eigenvector having a larger eigenvalue as the transformation axis. This guarantees that the transformed data suffers minimum loss as compared to the original.

Expert systems [29], artificial neural networks (ANN) [30], and fuzzy logic [31] are some of the classifiers that have been used to detect faults in rotating machines in past years. Moreover, some shortcomings like the low convergence rate, evident over-fitting, and local optimal solution are easy to include in these methods, limiting their application in real-world scenarios. Furthermore, when these methods are employed to analyze insufficient training sample, they have poor generalization. The Decision Tree [32] is a supervised machine learning algorithm that is efficient and takes less time to compute. It can also make a reliable judgement even for a limited number of data samples while simultaneously having outstanding generalization performance. Many researchers have exploited the virtues of a Decision tree to classify faults in various mechanical parts such as gears, bearings, and rotor, etc $[33,34]$. 
The following are the goals of this paper:

1. To identify hidden fault signatures, the raw vibration signal was denoised using a state-of-the-art MODWT signal processing approach.

2. Various time-domain statistical features were extracted.

3. To reduce the dimensionality of the feature set PCA is used and to prepare the dataset for AI utilization.

4. Fault classification and performance comparison with and without PCA datasets using Decision Tree.

The paper is arranged as follows. Sections 2 and 3 detail the experimentation setup and methodology used, as well as the results respectively. Finally, In Section 4, the conclusion will be presented.

\section{EXPERIMENTATION AND METHODOLOGY}

This section describes the experimental setup utilized in the research work as well as the approach used to extract relevant information from machine vibration signals.

\subsection{Experimental Setup}

A gearbox diagnostic simulator (GDS) was utilized to simulate gear faults. Figure 1 depicts the investigation's experimental setup. A healthy gear as well as three gears having root crack defects of magnitude 30 percent, 50 percent, and 70 percent were considered, and by utilizing wire electrical discharge machining (Wire EDM), these defects were generated. Figure 2 depicts all the gears used for the investigation. Table 1 lists the nomenclature used for all the gears.

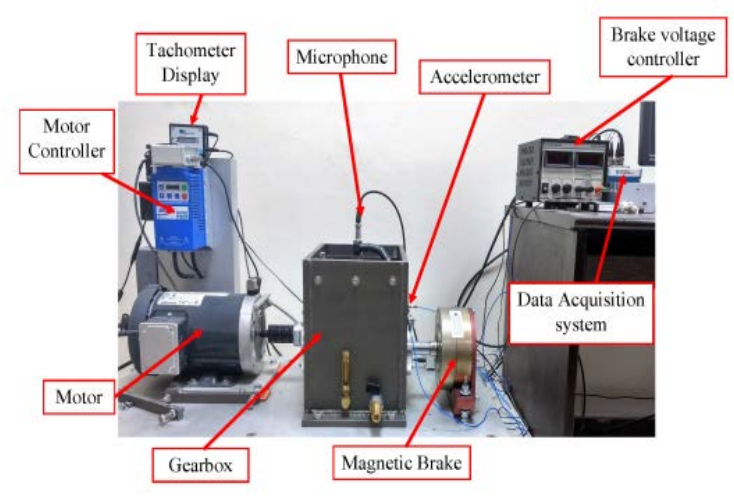

Figure1:Test rig used for experiment
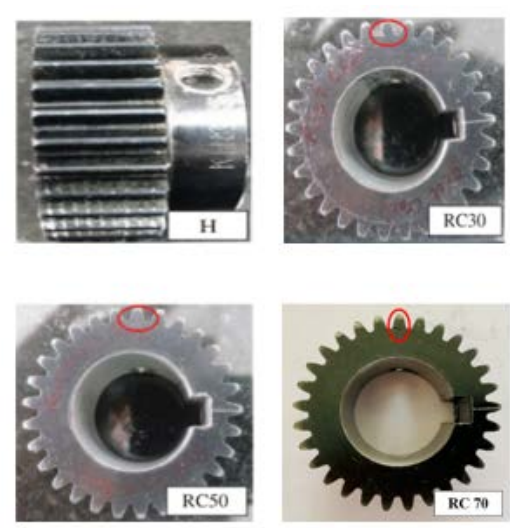

Figure 2:Gears with different health conditions

Table 1:Defect Description

\begin{tabular}{cc}
\hline $\begin{array}{c}\text { Defect } \\
\text { Description }\end{array}$ & Nomenclature \\
\hline Healthy & RC0 \\
Root crack of & RC30 \\
30\% & \\
Root crack of & RC50 \\
$50 \%$ & \\
Root crack of & RC70 \\
$70 \%$ & \\
\hline
\end{tabular}




\subsection{Methodology}

Figure 3 depicts the systematic process used in fault detection of gears with different magnitudes of defects.

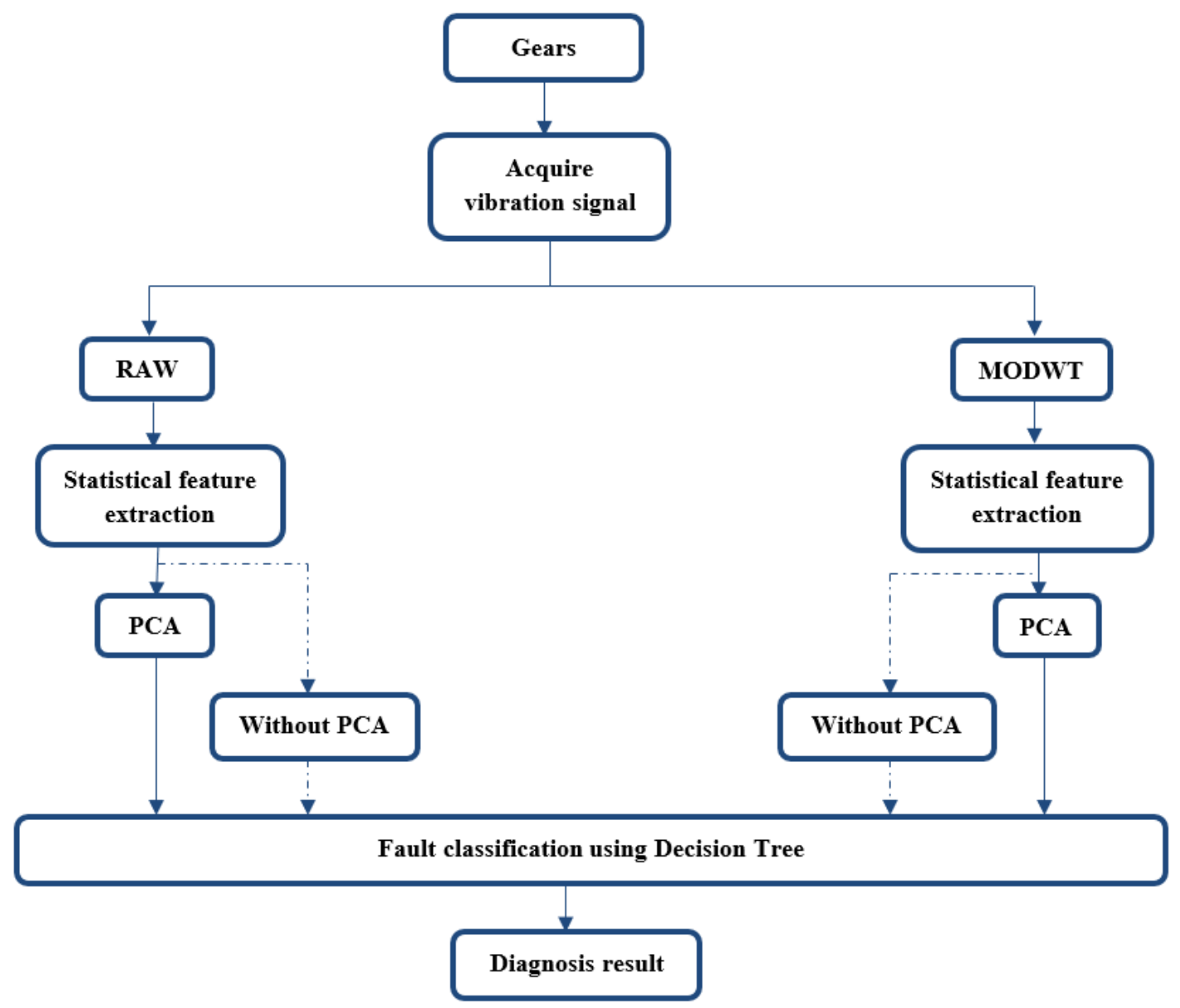

Figure 3: Experimental Methodology

\subsubsection{Data Acquisition}

The accelerometer was mounted on the bearing housing and utilized to capture the vibration signal for $13 \mathrm{~Hz}$ at $50 \%$ loading. The raw data from the gearbox was acquired using a data acquisition system (DAQ) with a sampling frequency of $25600 \mathrm{~Hz}$. Five repetitions of each gear condition were made in order to provide a sufficient quantity of signals for training and testing.

\subsubsection{Signal processing}

The processing was implemented on the Mathworks MATLAB release 2020b. The raw vibration signature was processed using a MODWT signal processing approach. Because the theoretical gear mesh frequency and its harmonics fit within the range of the selected decomposition level, the level of decomposition was chosen as 4 and 'sym4' as the mother wavelet.

\subsubsection{Feature Extraction}

A total ofeleven-time domain statistical features were utilized for fault diagnosis in case of raw and MODWT processed data viz. Margin factor, Skewness, Shape factor, Kurtosis, Peak to Peak, RMS, Impulse factor, Standard deviation, Crest factor, Energy, and Mean. 


\subsubsection{Dimension Reduction}

PCA then compresses the dimensions of the extracted feature datasets, maintaining as much statistical information as possible. It works by projecting a set of data onto a lowerdimensional subspace. The data is represented with no or very little collinearity in this reduced subspace.

\subsubsection{Classification and Performance Evaluation}

The resulted Principal Component having the highest eigenvalue is selected and utilized as an input for the Decision Tree for fault classification. The hold-out method was used for splitting the data into training and test set which is $70 \%$ for training and $30 \%$ for testing.

Also, the extracted features without reducing their dimensionality i.e without PCA application are fed as an input to the decision tree for fault classification and comparing the performance with processed features with PCA.

The confusion matrix along with the Receiver operating characteristic curve (ROC curve) were plotted to evaluate and compare the performance.

\section{RESULTS AND DISCUSSION}

\subsection{Signal processing}

The data was collected for about twenty-five times for each gear health condition at $13 \mathrm{~Hz}$ speed and $50 \%$ loading condition. Following that, the data was processed using the Maximal overlap discrete wavelet transform (MODWT) signal processing approach, as described in the methodology section. The amplitude trend of Raw Vibration data and MODWT denoised data with fault advancement is depicted in Figures 4 and 5, respectively. Figures reveal that there is no substantial indication of developing defects in raw vibration data until the fault development approaches RC70 and a few spikes are visible in the RC70 case. However, spikes are more evident in the denoised data even in the early stages of fault progression, i.e. RC30 and RC50, with the RC70 being the most noticeable

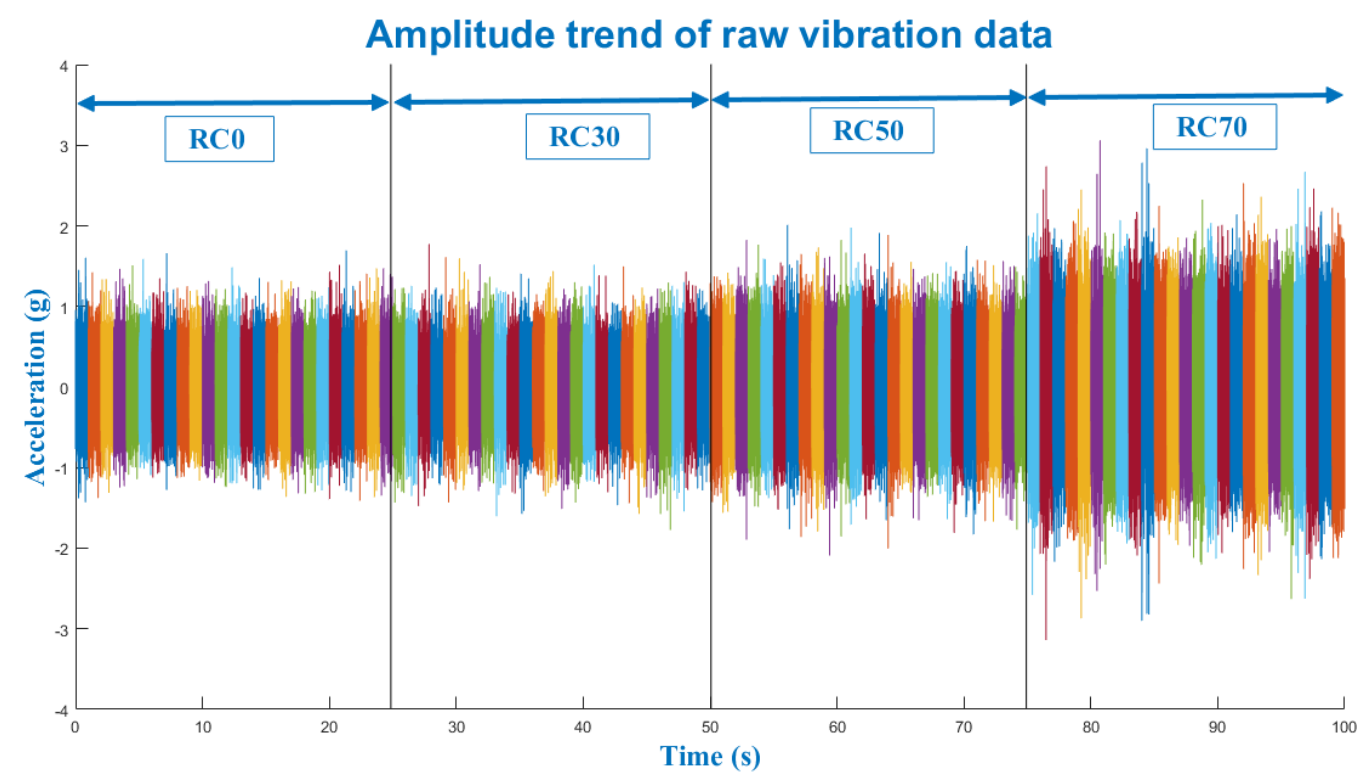

Figure 4. Amplitude trend for Raw vibration data with fault progression 


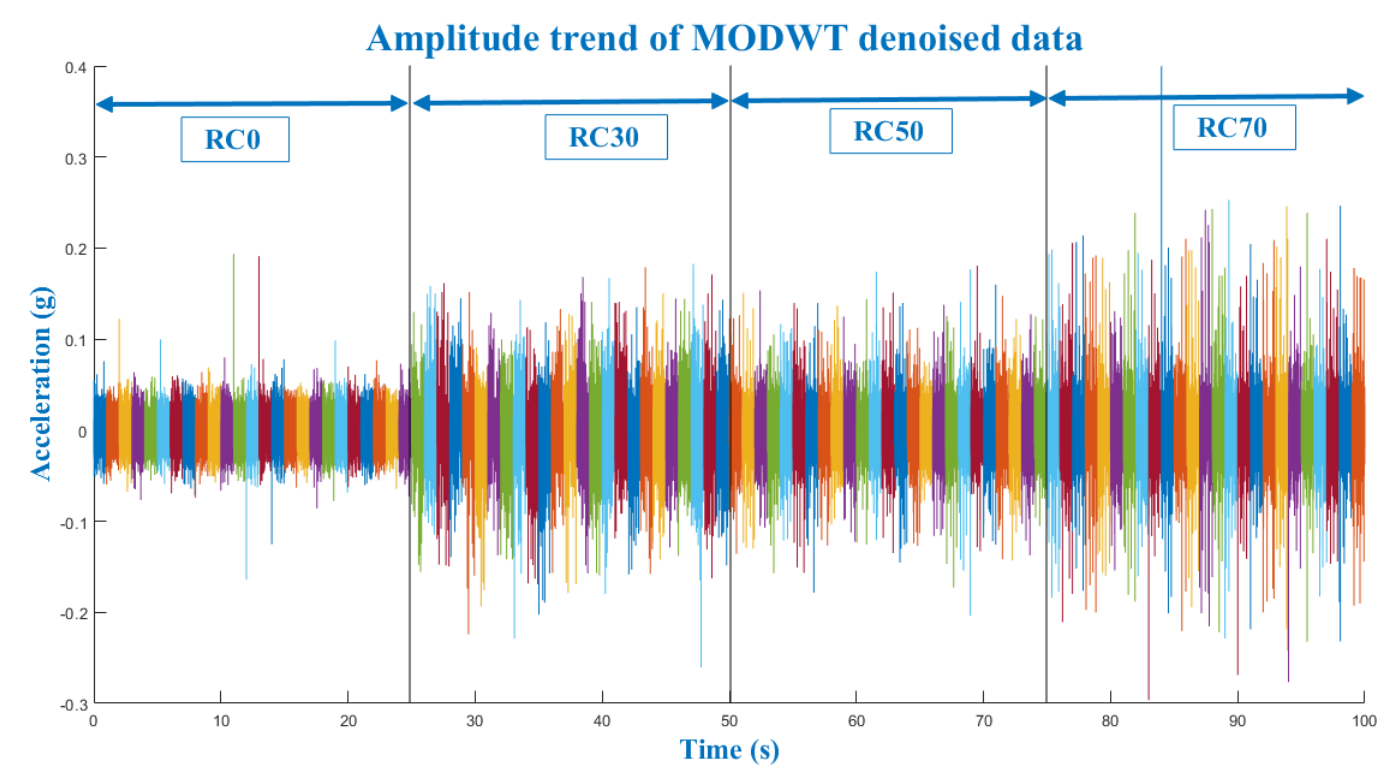

Figure 5. Amplitude trend for MODWT denoised data with fault progression

\subsection{Dimensionality Reduction of Datasets}

The PCA analysis of the Raw and MODWT denoised dataset is depicted in Figures 6 and 7 respectively.

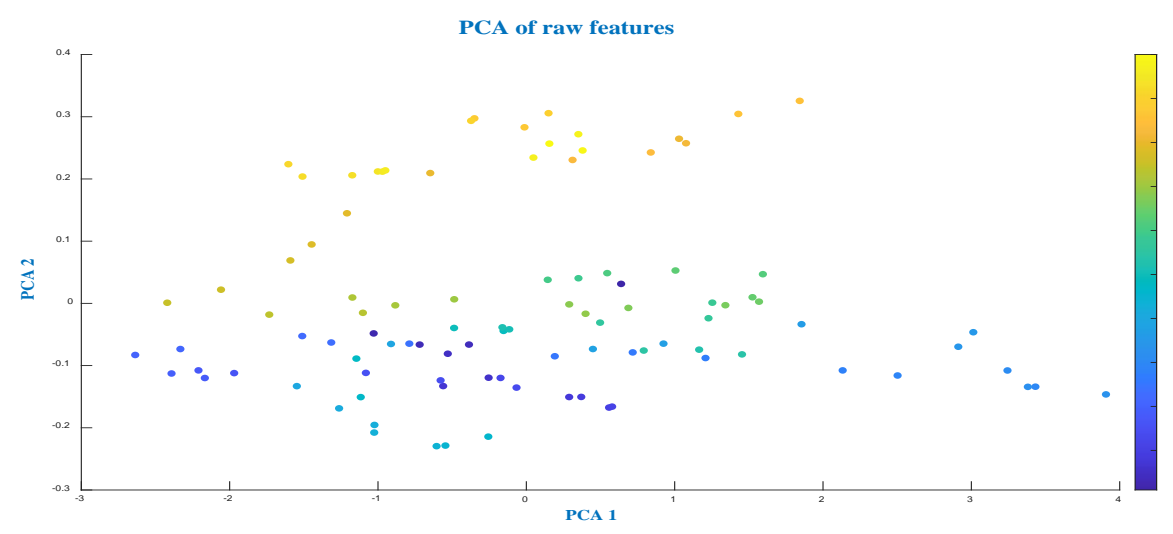

Figure 6: PCA analysis of Raw features

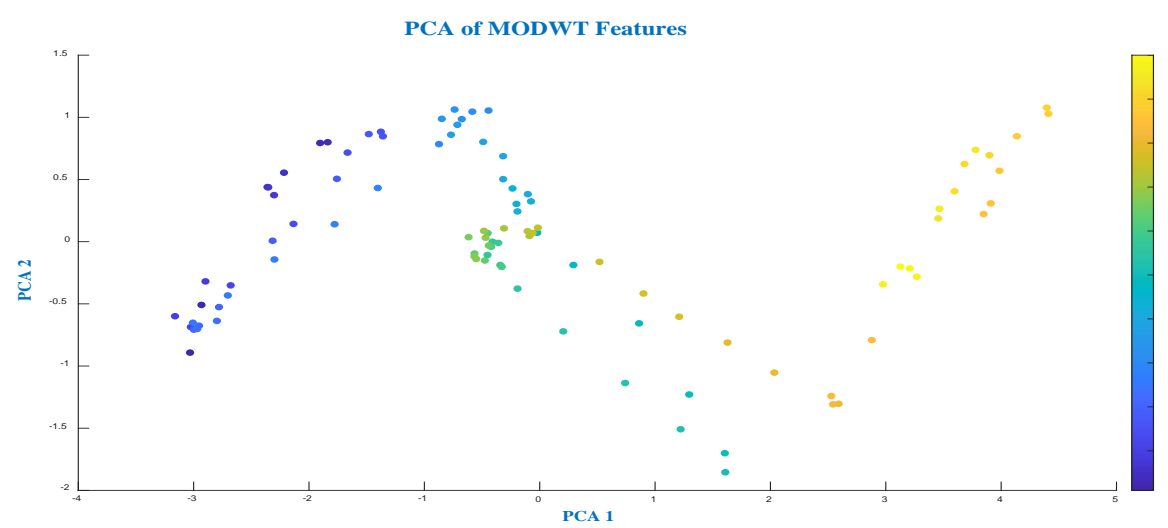

Figure 7: PCA analysis of MODWT denoised features 
PCA1 has higher variability than PCA2, as illustrated in the figures. As a result, it was chosen for both the datasets and fed into the Decision tree for fault classification.

\subsection{Classification Results}

\subsubsection{Classification Accuracies}

Figures 17 and 18 demonstrate the confusion matrix for raw data without PCA and with PCA Application, respectively

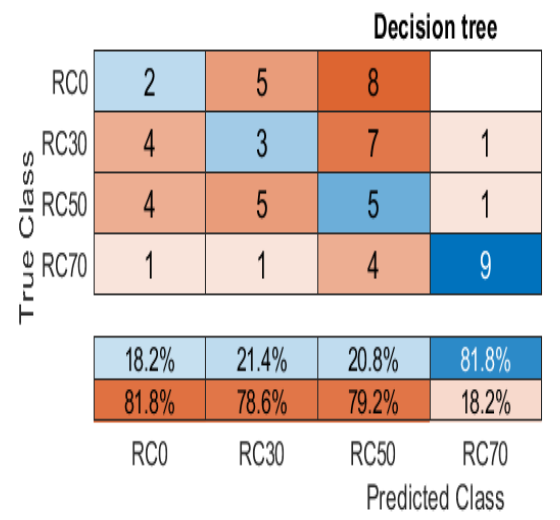

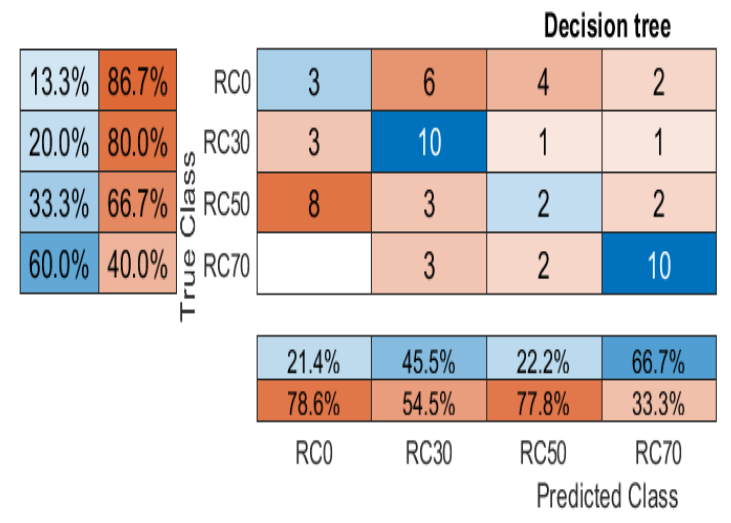

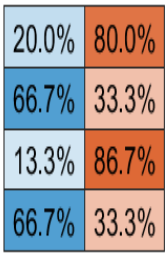

Figure 8: Confusion matrix of raw data without PCA Figure 9: Confusion matrix raw data with PCA

In the case of raw data without PCA application, the classification accuracy is $31.67 \%$ which is very less. Also, the labels i.e different gear health conditions are not categorized by the model. The highest classified label is RC70 because there is a substantial increase in its value, and the rest of the labels are misclassified. When PCA results were used, the accuracy increased to $41.67 \%$, although it is still not up to the mark but higher than when PCA was not applied. The maximum classified labels are RC30 and RC70. When dealing with raw data, the classifier fails to distinguish between the various gear health situations.

Figures 10 and 11 depict the confusion matrix for MODWT denoised data without PCA and with PCA application, respectively.
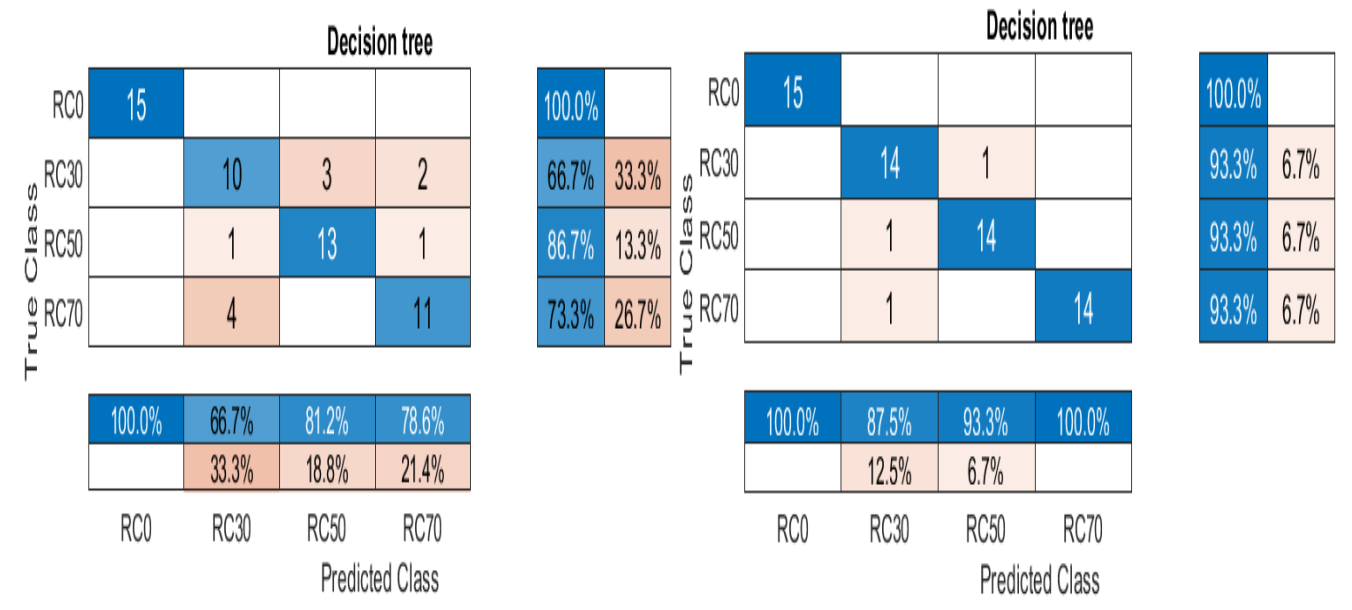

Figure 10: Confusion matrix of Figure 11: Confusion matrix denoised denoised data without PCA data with PCA

For MODWT, the classification accuracy without PCA is $81.67 \%$ which is better than the accuracy of the raw dataset without PCA. After PCA, the accuracy increased to 95\% which is way more than the accuracy of raw data set with PCA and also higher than the MODWT 
dataset without PCA. After denoising, the different gear conditions are well categorized by the model. After dimensionality reduction with PCA, the models performed better and achieved higher accuracy as illustrated in table 2.

Table 2: Accuracy of datasets with and without PCA application

\begin{tabular}{cccc}
\hline $\begin{array}{c}\text { Raw } \\
\text { accuracy } \\
\text { Without PCA }\end{array}$ & $\begin{array}{l}\text { Raw } \\
\text { accuracy with } \\
\text { PCA }\end{array}$ & $\begin{array}{l}\text { MODWT } \\
\text { accuracy } \\
\text { without PCA }\end{array}$ & $\begin{array}{l}\text { MODWT } \\
\text { accuracy with } \\
\text { PCA }\end{array}$ \\
\hline $31.67 \%$ & $41.67 \%$ & $81.67 \%$ & $95 \%$
\end{tabular}

\subsubsection{Receiver operating characteristic curve (ROC curve) Results}

The ROC curve for raw data without PCA and with PCA is depicted in Figures 12 and 13 respectively
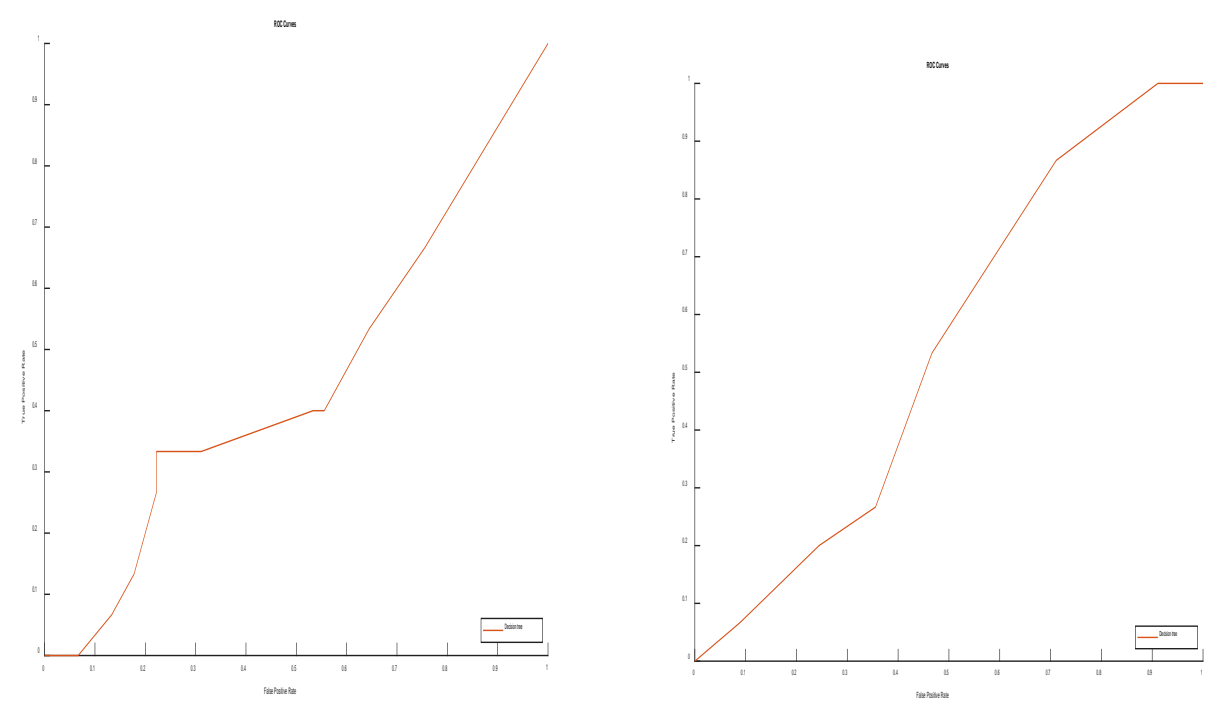

Figure 12: ROC Curve of raw data without PCA Figure 13: ROC Curve matrix raw data with PCA

ROC curve revealed that the PCA applied data has a higher Area under the curve (AUC) than the data without PCA which indicates that it is more closer to the true positive rate but its AUC is around 0.5 which represents that its ROC curve represents random chance.
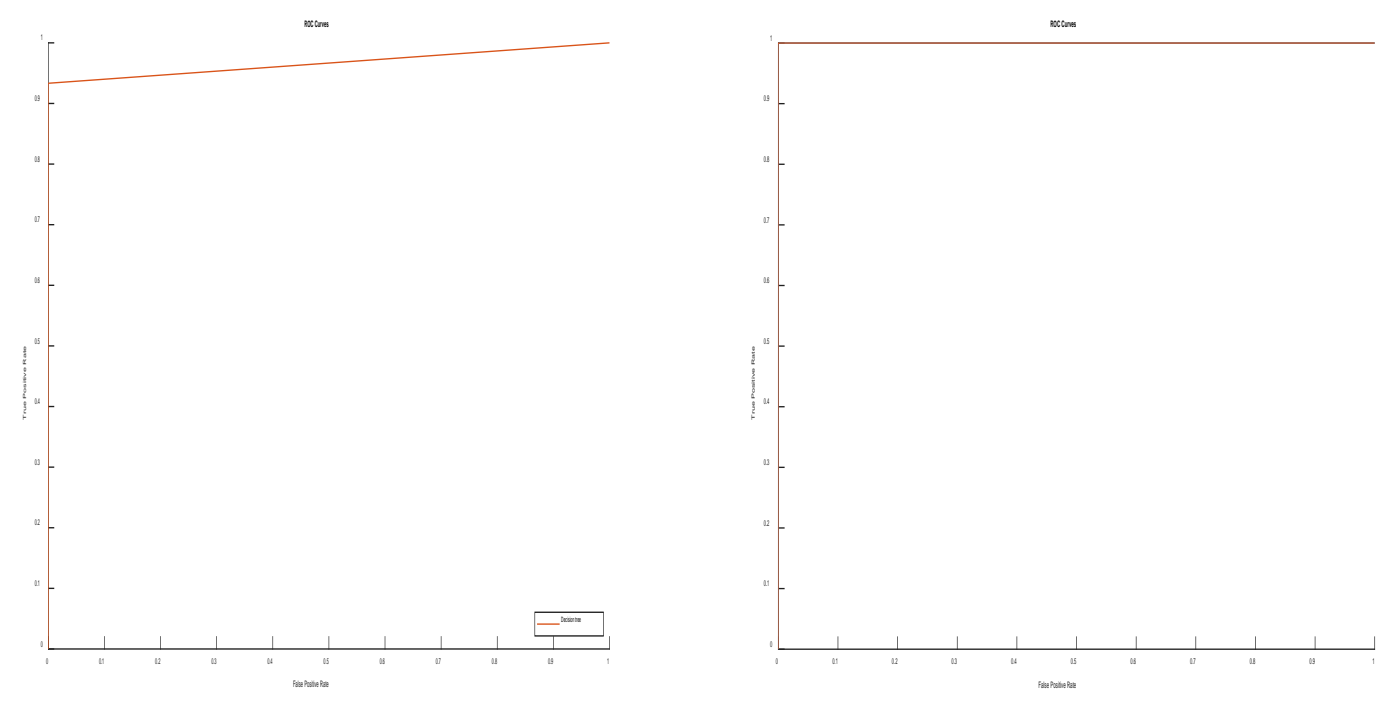

Figure 14: ROC Curve of raw data without PCA Figure 15: ROC Curve matrix raw data with PCA 
The classifier with PCA has a value of 1 for both the ROC and AUC curves, indicating that the classifiers can perfectly differentiate all the positive class values from the negative class values, as shown in the figure. The AUC without PCA is around 0.966, which is also good, and there is a fair chance that the classifier will be able to discriminate positive from negative class values.

\section{CONCLUSION}

This paper presents an efficient gear fault detection approach for categorizing various gear health conditions. The Maximal overlap discrete wavelet transform (MODWT) approach was used to process the raw vibration signals. Time-domain statistical characteristics were identified, and because it includes large dimensionality of data and redundant information, the paper uses principal component analysis (PCA) to reduce the dimensions of the extracted features and the resulting principal component being used as input to the classifiers and compares the results with those obtained without the use of PCA.

The classification results have shown that the methodology adopted to process the signal using MODWT and thereafter reducing the dimensionality of the dataset with PCA outperformed the raw results and the results without using PCA. So, it can be concluded from the findings that the dimensionality reduction in the Fault classification and diagnosis of gears is a crucial step as it yields better results.

\section{References}

1. F. Chaari, T. Fakhfakh, M. Haddar, Analytical modelling of spur gear tooth crack and influence on gearmesh stiffness, Eur. J. Mech. A/Solids. 28 (2009) 461-468. doi:10.1016/j.euromechsol.2008.07.007.

2. Renping Shao, Xinna Huang, Hongyu Liu, Fault detection and diagnosis of gear system based on higher order cumulants, Chin. J. Mech. Eng. 44 (6) (2008) 161-168

3. Bansal S, Sahoo S, Tiwari R, Bordoloi DJ. Multiclass fault diagnosis in gears using support vector machine algorithms based on frequency domain data. Measurement. 2013 Nov 1;46(9):3469-81.

4. F. Marquez, A. Tobias, J. Perez, M. Papaelias, Condition monitoring of wind turbines: techniques and methods, Renew. Energy 46 (2012) 169 e178.

5. W.X. Yang, C. Little, R. Court, S-Transform and its contribution to wind turbine condition monitoring, Renew. Energy 62 (2014) 137e146.

6. Wegerich SW, Wilks A, Pipke R. Nonparametric modeling of vibration signal features for equipment health monitoring. InProceedings of the IEEE aerospace conference 2003 Mar 8 (Vol. 7, pp. 3113-3121).

7. A.K.S. Jardine, D. Lin, D. Banjevic, A review on machinery diagnostics and prognostics implementing condition-based maintenance, Mech. Syst. Signal Process. 20 (2006) 1483-1510. doi:10.1016/j.ymssp.2005.09.012.

8. Terry, B, Lee, S. What is the prognosis on your maintenance program.Eng Min J 1995; 196: 32.

9. Dong ZH, Zhi-peng FE. Application of iterative generalized short-time Fourier transform to fault diagnosis of planetary gearboxes. 2017;39(4):604-10.

10. Wang LH, Zhao XP, Wu JX, Xie YY, Zhang YH. Motor fault diagnosis based on short-time Fourier transform and convolutional neural network. Chinese Journal of Mechanical Engineering. 2017 Nov 1;30(6):1357-68. 
11. Hoseinzadeh MS, Khadem SE, Sadooghi MS. Modifying the Hilbert-Huang transform using the nonlinear entropy-based features for early fault detection of ball bearings. Applied Acoustics. 2019 Jul 1;150:313-24.

12. Thakker HT, Dave V, Vakharia V, Singh S. Fault diagnosis of ball bearing using Hilbert Huang transform and LASSO feature ranking technique. InIOP Conference Series: Materials Science and Engineering 2020 May 1 (Vol. 841, No. 1, p. 012006). IOP Publishing.

13. Hartono D, Halim D, Wyn Roberts G. Gear fault diagnosis using an improved reassigned smoothed pseudo Wigner-Ville distribution. Cogent Engineering. 2018 Jan 1;5(1):1436928.

14. Sharma RR, Kalyani A, Pachori RB. An empirical wavelet transform-based approach for cross-terms-free Wigner-Ville distribution. Signal, Image and Video Processing. 2020 Mar;14(2):249-56.

15. Chen B, Shen B, Chen F, Tian H, Xiao W, Zhang F, Zhao C. Fault diagnosis method based on integration of RSSD and wavelet transform to rolling bearing. Measurement. 2019 Jan 1;131:400-11.

16. Malla C, Rai A, Kaul V, Panigrahi I. Rolling element bearing fault detection based on the complex Morlet wavelet transform and performance evaluation using artificial neural network and support vector machine. Noise \& Vibration Worldwide. 2019 Oct;50(9-11):313-27.

17. S. Mallat, A Wavelet Tour of Signal Processing, second ed., Academic, Beijing, 2003.

18. Chen J, Zi Y, He Z, Wang X (2013) Adaptive redundant multi-wavelet denoising with improved neighboring coefficients for gearbox fault detection. Mech Syst Signal Process 38(2):549-568.

19. H.L. Sun, Y.Y. Zi, Z.J. He, J. Yuan, Customized multiwavelets for planetary gearbox fault detection based on vibration sensor signals, Sensors 13 (2013) 1183e1209.

20. J. Wu and C. Hsu, Fault gear identification using vibration signal with discrete wavelet transform technique and fuzzy-logic inference, Expert Systems with Applications, 903-995.

21. J. Wu, C. Hsu and G. Wu, Fault gear identification and classification using discrete wavelet transform and adaptive neuro-fuzzy inference, Expert Systems with Applications, Volume 36, Issue 3, Part 2, Apr. 2009, Pages 6244-6255.

22. N. Saravanan and K.I. Ramachandran, Incipient gearbox fault diagnosis using discrete wavelet transform (DWT) for feature extraction and classification using artificial neural network (ANN), Expert Systems with Applications, Volume 37, Issue 6, Jun. 2010, Pages 4168-4181.

23. Y. Yang, Y. He, J. Cheng and D. Yu, A gear fault diagnosis using Hilbert spectrum based on MODWPT and a comparison with EMD approach, Measurement, Volume 42, Issue 4, May. 2009, Pages 542-551.

24. 40. P. Shan and M. Li, Nonlinear Time-Varying Spectral Analysis: HHT and MODWPT, Mathematical Problems in Engineering, Volume 2010, Article ID 618231, 14 pages, Mar. 2010.

25. Shensa MJ. The discrete wavelet transform: wedding the à trous and Mallat algorithms. IEEE Trans Signal Process 1992;40(10):2464-82.

26. Hafez AG, Ghamry E. Geomagnetic sudden commencement automatic detection via MODWT. IEEE Trans Geosci Remote Sens 2013;51(3):1547-54.

27. Shao R, Hu W, Wang Y, Qi X. The fault feature extraction and classification of gear using principal component analysis and kernel principal component analysis based on the wavelet packet transform. Measurement. 2014 Aug 1;54:118-32. 
28. Bao Bisai, Xiaojun Lou, Application of principal component analysis in target recognition algorithm of seismic signals, J. Huazhong Univ. Sci. Technol. Nat. Sci. 40 (7) (2012) 24-28.

29. Li W, Shi T, Liao G, Yang S. Feature extraction and classification of gear faults using principal component analysis. Journal of Quality in Maintenance Engineering. 2003 Jun 1.

30. D. Ma, Y. Liang, X. Zhao, et al., Multi-BP expert system for fault diagnosis of power system, Eng. Appl. Artif. Intell. 26 (2013) 937-944.

31. S.S. Tayarani-Bathaie, Z.N.S. Vanini, K. Khorasani, Dynamic neural network-based fault diagnosis of gas turbine engines, Neurocomputing, 125 (2014) 153-165.

32. V. Muralidharan, V. Sugumaran, Rough set based rule learning and fuzzy classification of wavelet features for fault diagnosis of monoblock centrifugal pump, Measurement 46 (2013) 3057-3063.

33. W. Sun, J. Chen, J. Li, Decision tree and PCA-based fault diagnosis of rotating machinery, Mech. Syst. Signal Process. $21 \quad$ (2007) 1300-1317. doi:10.1016/j.ymssp.2006.06.010.

34. V. Sugumaran, K.I. Ramachandran, Automatic rule learning using decision tree for fuzzy classifier in fault diagnosis of roller bearing, Mech. Syst. Signal Process. 21 (2007) 2237-2247. doi:10.1016/j.ymssp.2006.09.007

35. N.R. Sakthivel, V. Sugumaran, S. Babudevasenapati, Vibration based fault diagnosis of monoblock centrifugal pump using decision tree, Expert Syst. Appl. 37 (2010) 4040-4049. doi:10.1016/j.eswa.2009.10.002 